\title{
« Mystères » bouddhiques. La théâtralisation des rituels tibétains par les voyageurs au début du $\mathrm{XX}^{\mathrm{e}}$ siècle
}

Buddhist "mystery plays." Early 20th century travellers and Tibetan rituals as theatre

Samuel Thévoz

\section{OpenEdition}

\section{Journals}

\section{Electronic version}

URL: https://journals.openedition.org/emscat/2622

DOI: 10.4000/emscat.2622

ISSN: 2101-0013

Publisher

Centre d'Etudes Mongoles \& Sibériennes / École Pratique des Hautes Études

\section{Electronic reference}

Samuel Thévoz, " « Mystères » bouddhiques. La théâtralisation des rituels tibétains par les voyageurs au début du XXe siècle", Études mongoles et sibériennes, centrasiatiques et tibétaines [Online], 46 | 2015, Online since 10 September 2015, connection on 13 July 2021. URL: http://journals.openedition.org/ emscat/2622 ; DOI: https://doi.org/10.4000/emscat.2622

This text was automatically generated on 13 July 2021.

(c) Tous droits réservés 


\section{« Mystères » bouddhiques. La théâtralisation des rituels tibétains par les voyageurs au début du $\mathrm{XX}^{\mathrm{e}}$ siècle}

Buddhist "mystery plays." Early 20th century travellers and Tibetan rituals as theatre

Samuel Thévoz

\section{Approcher les rituels tibétains à travers l'expérience des voyageurs}

$1 \quad$ Il y a à peine plus d'un siècle, les explorateurs du Tibet se faisaient les premiers témoins des rituels pratiqués dans ce pays encore mal connu ${ }^{1}$. Les voyageurs dont il sera question ici sont Sven Hedin (1910), Henri d'ollone (1911), Jacques Bacot (1909 et 1912) et Alexandra David-Néel ([1912] 1975). Pour apprécier la valeur de ces premières rencontres avec les formes cultuelles tibétaines, les sciences humaines offrent aujourd'hui plusieurs approches que j'esquisserai selon trois « voies » majeures.

2 La première cherche à accéder à une connaissance de l'autre en dépassant les incompréhensions de la rencontre interculturelle, les biais idéologiques et les torsions que l'écriture impose à l'expérience vécue. C'est l'approche de l'ethno-histoire ou de l'anthropologie historique ${ }^{2}$. Dans cette perspective, les textes des voyageurs traduisent un état de la culture tibétaine et offrent au spécialiste des connaissances positives sur la société étudiée à travers leurs descriptions de différents aspects des rites tibétains : cérémonies de Nouvel An, cérémonies officielles, danses 'cham, "théâtre sacré ", oracles.., etc.

3 Une seconde voie cherche à replacer ces textes dans le contexte épistémologique de leur temps. C'est l'approche de l'histoire des sciences. On soulignera alors que ces 
voyages, menés dans les années 1910, s'effectuent à un moment-clé des sciences des religions: les notions de rite et de sacré retiennent alors l'attention des sciences humaines alors en voie d'institutionnalisation. Émile Durkheim amorçait un débat qui se poursuit dans les réflexions de Marcel Mauss, de Robert Hertz et de Lucien LévyBruhl (Tarot 1999, 2003; Keck 2008). Tout en portant un intérêt remarquable aux formes rituelles tibétaines, les voyageurs montrent combien ils étaient tributaires des champs de questionnement de leurs contemporains (Thévoz 2010) et participent du contexte épistémologique de leur époque. En livrant dans leurs récits de voyage ou dans leur correspondance des témoignages à caractère ethnographique, ils offrent par ailleurs une source majeure d'information pour l'anthropologie et la tibétologie naissantes autant qu'un réservoir d'images pour l'imaginaire collectif qui tient, jusqu'à aujourd'hui, les formes religieuses tibétaines pour les manifestations les plus terrifiantes du mysterium tremendum ${ }^{3}$, notion-clé des études sur le « sacré » du début du $\mathrm{XX}^{\mathrm{e}}$ siècle. Il est utile de rappeler par exemple que Rudolf Otto, pour qui le "mystère " représente «ce qui est absolument en dehors du domaine des choses [...] " familières » " (Otto [1917] 2001, p. 58) ${ }^{4}$, définissait le mysterium tremendum comme le "sentiment du mystère qui fait frissonner » (ibid., p. 36) dont la manifestation la plus aiguë est l'« horreur sacrée »:

Le sentiment d'horreur n'est pas la crainte naturelle et ordinaire, c'est déjà la première apparition et comme un vague pressentiment du mystérieux sous la forme rudimentaire $\mathrm{du}$ "sinistre », la première évaluation d'après une catégorie qui ne se trouve pas dans le domaine commun et naturel et ne s'applique pas à quelque chose de naturel. (ibid., p. 40)

Une troisième voie envisage les textes des voyageurs sous l'éclairage de l'histoire du regard porté sur le Tibet, c'est-à-dire celle de l'imagologie ou de l'histoire des représentations. $\mathrm{Au} \mathrm{XIX}^{\mathrm{e}}$ siècle, toute manifestation religieuse au Tibet était perçue à l'aune de la notion de "lamaïsme » dont la notion de "rite " était constitutive ${ }^{5}$. Ainsi n'est-il pas inutile de préciser que, à l'heure où les voyageurs de la première décennie $\mathrm{du} \mathrm{XX}^{\mathrm{e}}$ siècle partaient pour le Tibet, la religion des "barbares de l'Ouest » était approchée selon une axiologie négative. En 1847, l'indianiste Théodore Pavie, dans son manifeste en faveur des études tibétaines assurées alors par Philippe Édouard Foucaux à l'École des langues orientales de Paris depuis 1842, décrivait ainsi le bouddhisme tibétain en s'appuyant sur les chroniques chinoises, les récits des missionnaires et les quelques témoignages de voyageurs comme celui du père Huc ([1850] 2001). Pavie décrivait ainsi la religion tibétaine :

La période thibétaine [du bouddhisme], ou le lamaïsme, se continue encore et montre la religion de Bouddha passée dans les mœurs des peuples, mais immobile, stationnaire et languissante. [...] L'intronisation des lamas au Thibet date du XIII' siècle ; voilà six cents ans qu'ils s'y maintiennent dans une condition tout-àfait passive. [...] Depuis la fondation du bouddhisme, le chef de la doctrine a toujours été considéré comme une incarnation du législateur divinisé ; au Thibet, cette croyance a conduit les fidèles au fétichisme le plus outré. (Pavie 1847, pp. 46-47)

Dans la perspective philologique de Pavie, le dogme de l'Église tibétaine, à l'origine du bouddhisme dégénéré dit du Nord, se fonde sur des livres dont la lettre l'emporte sur l'esprit et conduit à un ritualisme où le geste sans signification tend à la pensée magique, voire à la sorcellerie :

L'ensemble des ouvrages bouddhiques conservés dans les états (sic) du lama se divise en deux catégories. La première comprend le Kan-djour ou préceptes traduits, 
collection de cent volumes qui renferme le rituel proprement dit. Le thibétain étant la langue de la liturgie, le latin de la religion de Bouddha, ce rituel a été adopté par tous les couvens bouddhiques de la Tartarie et de la Chine [...]. Dans la seconde, appelée Stan-djour, sont contenues les instructions traduites, les incantations, les formules de malédiction et d'exorcisme. (ibid., p. 45)

L'auteur résume, en le déplorant, l'essor de ce culte marqué du sceau de la décadence :

Après s'être manifestée comme une loi d'émancipation pour toutes les castes opprimées et de réhabilitation pour toutes les créatures, après avoir pris la forme d'une religion consacrée par un rituel, cette croyance égarée qui vint aboutir au dogme du vide et du néant, à l'absorption pure et simple dans le grand être universel, recruta, chemin faisant, les superstitions répandues dans toute l'Asie. (ibid.)

7 Dans la réduction des paroles primitives du Bouddha à un ensemble constitué de rites, Pavie reconnaît le " culte du néant " qu'est alors le bouddhisme pour l'Occident (Droit 1997 et App 2012). S'appuyant sur cet état général de la réception du bouddhisme tibétain, l'histoire des représentations, cherchant avant toutes choses à repérer des permanences et des phénomènes de longue durée, soulignera la survivance tout au long $\mathrm{du} \mathrm{XX}^{\mathrm{e}}$ siècle des avatars du «lamaïsme » sous les traits notamment du «mythe » de Shangri-La (Bishop 1989, Lopez [1998] 2003, Thévoz 2011). En revanche, cette approche ne s'emploiera pas à faire apparaître les singularités propres à chaque voyageur et, partant, de rendre visibles les changements dont ceux-ci sont porteurs, à leur échelle, dans la perception des rituels tibétains.

\section{Drames sacrés : de rituel en mystère}

Ces approches, on le voit, ont toutes leur légitimité propre. Néanmoins, la lecture que je propose aimerait être plus minutieusement attentive aux dimensions littéraires de ces textes. Dans cette perspective, elle procède, pour commencer, au repérage des lieux communs aux voyageurs lorsqu'ils décrivent les rituels tibétains, indépendamment de la variété, voire de l'incommensurabilité, des phénomènes que ceux-ci recensent dans leurs récits. Comme nous le verrons, il ressort que les voyageurs décrivent les formes cultuelles observées en des termes dramaturgiques, plutôt que directement liturgiques par exemple. De toute évidence, ils appliquent à la culture tibétaine un schème d'intelligibilité particulièrement efficace dans le contexte historique et culturel qui était le leur. Toutefois, ce schème ne se réduit pas à un modèle explicatif préexistant à la rencontre et à l'expérience de voyage. Au contraire, il facilite potentiellement l'interaction et ouvre la possibilité d'une rencontre interculturelle in situ ${ }^{6}$ : c'est là le deuxième temps de l'analyse, qui permettra de cerner les usages du " prisme » théâtral propres à chacun des voyageurs.

9 Si l'on y regarde de plus près, la référence dramaturgique à laquelle les voyageurs rapportent unanimement les rites de la religion tibétaine se cristallise autour de la notion spécifique de «mystère». De par les liens consubstantiels entre théâtre et religion qu'elle suppose à leurs yeux, la forme du « drame liturgique » médiéval (et ses dérivés : "mystère », «miracle», "moralité») semble s'imposer comme référence hautement signifiante aux voyageurs.

10 En effet, ces arpenteurs d'un monde inconnu, pour être des explorateurs, n'en sont pas moins imprégnés de la culture lettrée de la fin du XIX ${ }^{\mathrm{e}}$ siècle. Rappelons à ce propos que, dans le sillage de Gaston Paris, la nouvelle école de philologie romane (Ridoux 
1996) redécouvre par les textes les formes de la dramaturgie médiévale (et notamment de la fin du Moyen Âge, où le théâtre religieux connaît son essor) ${ }^{7}$. De surcroît, la résurgence $d u$ "mystère " exerce son influence sur la dramaturgie symboliste du tournant du siècle ${ }^{8}$. Chez des auteurs comme Auguste Villiers de l'Isle-Adam, Stéphane Mallarmé, Maurice Bouchor, Maurice Maeterlinck ou Paul Claudel, le terme "mystère » est mis au service de "leur ambition de porter à la scène un monde spirituel et de donner une représentation élargie de la vie humaine, aussi bien objective que subjective " (Losco-Lena 2010, p. 17). Dans cette perspective, l'on explore, en dehors de toute orthodoxie religieuse et souvent même d'une quelconque obédience confessionnelle, les liens de la scène théâtrale avec l'« outre-monde » (Losco-Lena 2010, pp. 14-18). Le "mystère » appartient ainsi fortement à la culture des voyageurs au moment où ils prennent la direction du Tibet.

L'on sait que les "mystères " médiévaux mettaient traditionnellement en scène des épisodes bibliques ou hagiographiques (Pavis [1996] 2006, p. 225). Pour les Symbolistes, le «mystère " est revêtu d'une vocation sacrée qu'il ne possédait pas à l'origine. Les voyageurs, quant à eux, quand ils emploient le terme, ne se réfèrent pas plus rigoureusement que leurs contemporains dramaturges aux textes du XV siècle. Les spécificités dramaturgiques du genre et la scénographie en "décors simultanés » sont volontiers négligées. Une idée $\mathrm{du}$ "mystère " règne alors, diversement ajustée aux connaissances philologiques. Symétriquement, la notion de "mystère " permet aux voyageurs de regrouper sous une même catégorie des phénomènes culturels disparates aux yeux de l'ethnographe d'aujourd'hui : cérémonies religieuses, danses rituelles, drames à thème religieux, rites ésotériques, etc.

Il ressort clairement de leurs récits que les explorateurs témoignent du goût pour l'art médiéval porté alors par le romantisme hugolien et le wagnérisme (Losco-Lena 2010, pp. 113-150). Dès lors, aux similitudes que les voyageurs se sentent autorisés à observer entre les structures sociales et l'univers de croyances «pré-moderne» du Moyen Âge occidental et du Tibet lamaïque, s'adjoint ainsi un régime de comparaison portant sur les formes esthétiques et dramaturgiques. À propos des "mystères» tibétains, ils retiennent par exemple comme points de comparaison les circonstances de l'exécution du drame : l'on sait à ce propos qu'au Moyen Âge, les «mystères " transformaient, le temps de la représentation, programmée à l'occasion de fêtes religieuses, les enceintes des abbayes, les églises ou les lieux urbains ordinaires en « espaces théâtralisés ». Les voyageurs partagent en outre l'idée entretenue ultérieurement par la critique littéraire que ces représentations sont des événements "collectifs " pratiqués sur le mode du "rite» actualisant les "mythes" fondateurs pour le bénéfice et en présence de l'ensemble de la «cité » (Baumgartner 1988, pp. 150-155). Les voyageurs rapprochent également du "mystère» la mixité générique de certaines formes dramatiques tibétaines (alternance du drame sacré avec des mimes, des farces et des jongleries), leur dispositif dramaturgique (intervention d'un récitant extérieur à l'action; chants et psalmodies se superposant au jeu des acteurs, religieux et laïcs en partie amateurs), la durée de la représentation, enfin, s'étalant volontiers sur plusieurs jours (Baumgartner 1988, pp. 189-193).

Ces quelques ressemblances de famille entre la tradition chrétienne médiévale et les rituels tibétains tendent à motiver la référence au « mystère ». La tension qui sous-tend les usages de ce terme dans le discours des voyageurs est inhérente à son étymologie même: «du latin ministerium, office, acte. Ou selon une autre étymologie, du latin 
mysterium, mystère, vérité secrète. » (Pavis [1996] 2006, p. 225)9. On perçoit d'emblée combien, en utilisant un terme aux significations labiles, tout exogène soit-il au monde tibétain, les voyageurs, loin de se contenter de ramener les formes rituelles tibétaines aux formes culturelles européennes (Bishop 1989, pp. 154-155), font résonner la notion de « mystère » sur l'arrière-fond de la perception du « lamaïsme » en Occident. En effet, le terme relève simultanément de la praxis (" office, acte») et de la métaphysique (" vérité secrète »). Appliqué au monde tibétain, il peut donc se référer d'une part au rite fétichiste, issu d'une "croyance égarée ", administré voire manipulé par le clergé (Pavie), et d'autre part au "drame sacré", dans l'acception romantique et postromantique que lui donnaient les contemporains des voyageurs. Dans ce second sens, le « mystère » se rapproche des cultes à mystères antiques et, plus encore, de la notion de mysterium tremendum telle que la définissait Rudolf Otto à la même période (cité supra).

On se rend ainsi compte combien l'orbe des questions esthétiques circonscrites ici croise les problématiques épistémologiques évoquées au préalable, tout en portant au jour, nous aurons le loisir de le constater, les positions idéologiques à l'œuvre dans l'approche des rituels tibétains.

Ce «prisme» théâtral mène certes à de nombreuses apories épistémiques, car il demeure in fine une catégorie exogène et surplombante. Cependant, j'aimerais montrer qu'il comporte des vertus heuristiques essentielles pour penser la rencontre avec d'autres cultures, il y a un siècle comme aujourd'hui. En effet, il permet de saisir, pour autant qu'il ne se fige pas en système, une diversité d'aspects du monde rituel tibétain ; sous ce rapport, il traduit la complexité de l'expérience interculturelle. Il importe dès lors d'examiner les multiples actualisations de la perception et de la représentation des rituels sous les traits de drames auxquels les voyageurs se sont accordés à attribuer, à tort ou à raison, des valeurs sacrées. En effet, loin de se limiter à indiquer que, comme le veut une longue tradition critique (Thévoz 2012), le regard du voyageur sur l'altérité renvoie in fine à sa culture propre et n'élabore jamais que des fictions, la pluralité des modes de "théâtralisation" des rituels conduit à en évaluer les implications épistémologiques. Celles-ci exigent à leur tour d'être reversées au bénéfice d'un changement de paradigme dans l'histoire de la tibétologie que les approches méthodologiques énumérées en préambule ne suffisent seules à expliquer.

Les quelques éléments développés jusqu'ici, outre la mise au point méthodologique auxquels ils ont servi, autorisent à se faire une image de ce que les premiers explorateurs ont en tête lorsqu'ils abordent la question des pratiques religieuses au Tibet. Pratiquement contemporains par leur date de publication, les récits des voyageurs se rapportent à des voyages légèrement espacés dans le temps et témoignent d'une évolution rapide des représentations et des savoirs.

\section{Dans les coulisses d'une mise en scène (Tashilhunpo, début de saison 1907)}

17 L'explorateur suédois Sven Hedin rapporte son expédition de 1906-1908 dans le célèbre Transhimalaya. Upptäckter och äfventyr $i$ Tibet $^{10}$. Le récit est traduit en français en 1911 sous le titre Le Tibet dévoilé. Nul doute donc qu'en ouvrant l'ouvrage le lecteur parisien du début du siècle, sensible aux promesses du titre séduisant, n'ait été habité par la soif de découvrir à distance les grands espaces vierges inconnus du Tibet, sinon hanté par le désir de voir révélée une culture aux contours inquiétants. Arrivé en février 1907 au 
Tashilhunpo (Kra shi lhun po) de Shigatse ( $g$ Shis ka rtse), le célèbre monastère où siégeaient, traditionnellement, les panchen lamas, Hedin souligne qu'y règne "une impression de chose profondément mystérieuse et cachée " (Hedin 1910, p. 106), formule qu'il répète à l'envi. Ainsi le voyageur se fait-il fort d'aviver les aspirations de son lecteur quand il déclare: "pendant mes longues et fréquentes promenades, j'ai fréquemment assisté à de curieuses cérémonies » (ibid., p. 112).

Le « prisme » théâtral à l'œuvre dans son récit des célébrations du nouvel an tibétain (lo gsar), assimile explicitement les pratiques «lamaïques» au "mystère» médiéval. Observateur souverain placé sur « un balcon dominant la scène et la salle » du "vaste théâtre en plein air » et jouissant ainsi d'une vision panoramique en surplomb (ibid., p. 97), Hedin occupe une position symétrique au «Tachi-lama» (panchen lama) qui, depuis son " estrade » peut, "sans être exposé aux regards de la foule ", " tout voir » à la manière du prince dans les salles "à l'italienne ». Par cette référence au théâtre classique, Hedin ajoute au registre du «mystère » une dimension qui trahit quelque idéologie. En effet, cette position de pouvoir, qui le distingue des « six mille spectateurs entassés dans les galeries, sur les toits, sur les murs, sur les corniches", conduit l'explorateur-ethnographe à dominer le spectacle vivant comme s'il le contemplait à travers une vitrine de musée: "Un éblouissant chatoiement de couleurs; une exposition de costumes de l'Asie centrale. » Néanmoins, après cette phase d'exposition (le terme appartient lui-même à la terminologie dramaturgique), artificiellement statique, la musique qui enveloppe le drame accapare tous les sens du spectateur et semble le soustraire à ses capacités critiques :

Tout à coup, du haut des toits, tombe un long mugissement. Des lamas soufflent dans d'énormes conques ; c'est l'annonce du commencement de la fête, et aussitôt la foule pousse de longues acclamations. Puis, d'une galerie cachée sous d'immenses voiles noirs monte un chœur, grave et lent, d'une sublime beauté religieuse et d'une puissance pénétrante. Jamais je n'ai entendu chant plus émouvant. (ibid., p. 98)

L'orchestration musicale, constituée principalement d'instruments à vent (conques, trompes) et de percussions (tambours, cymbales), est amplifiée par le chœur. L'ensemble rappelle simultanément par sa «sublime beauté religieuse " et sa "puissance pénétrante » le protocole liturgique du culte catholique, à la manière d'un introït, et l'« art total » wagnérien fin de siècle, familier à l'explorateur scandinave. Les phases de la cérémonie se succèdent: d'abord processionnelle, elle laisse place à l'apparition d'un oracle masqué derrière le «rideau noir » qui constitue le seuil de la « scène » :

Un masque, nommé Argham, s'élance alors, en dansant et en tenant à bout de bras une soucoupe remplie de sang de chèvre. D'un mouvement brusque, il la renverse sur les marches, puis continue ses ébats, les deux bras tendus. (ibid., p. 98)

Loin de rendre manifeste le mysterium tremendum annoncé par le chœur et l'orchestre, le caractère horrifique de la transe rompt au contraire, dans l'esprit rationnel du voyageur, l'envoûtement provoqué par la musique et le conduit à se rabattre sur des savoirs préalables sur le lamaïsme: "Très certainement, cette cérémonie est une survivance de l'antique religion indigène. Le lamaïsme est pénétré d'éléments sivaïstes et de pratiques remontant aux temps pré-bouddhiques » (ibid.).

21 La mixité générique du «mystère » amplifie l'« impureté » (ibid., p. 106) des formes rituelles tibétaines. On décèle dans le discours du voyageur une vision anthropologique dont le paroxysme du drame fournit la justification : 
Au début, tambours, trompettes et cymbales jouent piano, mais peu à peu, le mouvement se précipite, les musiciens tapent à tour de bras et soufflent à perdre haleine: c'est alors une effroyable cacophonie, et, enivrés par ce bruit assourdissant, les danseurs se démènent comme des possédés. Cette frénésie gagne les spectateurs. Des pèlerins se lèvent, et, se tournant vers le Tachi-lama, se prosternent trois fois de suite. Entre tous, un vieillard du Tchang-tang, vêtu de peaux, est infatigable; sans répit, on le voit se jeter à terre. Absorbé dans cette mimique, il ne voit pas une peau d'orange, glisse et fait une culbute à la grande joie de ses voisins. (ibid., p. 98) participation collective: mise en scène et drame débordent le cercle restreint des acteurs pour contaminer l'ensemble de l'assemblée. Puis le ton de la description change au fur et à mesure que se déploie la scène. En effet, celle-ci tourne à la farce; dans cette comédie, l'orchestration n'est plus qu'une "effroyable cacophonie». Le savoir de l'ethnographe l'autorise à voir au-delà des apparences : il peut lire dans les traits de son visage l'origine du vieux nomade, mais il le soupçonne, de par son comportement, de mauvaise foi. Il ne voit pas derrière sa mimique autre chose que des gestes sans signification: tout dans ce spectacle se réduit à un simulacre. Si les notations du voyageur donnent matière à une ethnocritique, force est de reconnaître que les données « ethnographiques » - à l'évidence répondant à l'ethnologie de son temps sont entrelacées à l'axiologie de Hedin :

Un tel spectacle doit laisser une impression profonde sur les pèlerins. Après avoir été témoins de ces mascarades d'un symbolisme effrayant et de ces luttes contre les démons toujours terminées par leur défaite, ces simples regagnent leur désert, pénétrés de la toute-puissance de la religion et de la nécessité de son secours pour échapper aux maux qui menacent l'humanité souffrante. (ibid.)

23

Quand, au début du récit, Hedin partageait au lecteur ses propres émotions face au cérémonial («Jamais je n'ai entendu chant plus émouvant»), il soulignait les effets recherchés d'une mise en scène, tandis qu'in fine il fait voir comment l'observateur rationnel débusque ce stratagème religieux. Il ne met pas en doute la force de l'impression mais, contrairement aux fidèles, il a les moyens, par l'exercice de la raison critique, de se détacher de cette puissance. En définitive, la comparaison avec le "mystère " médiéval opère comme un repoussoir et marque un profond déséquilibre où la farce l'emporte toujours sur le drame sacré. Dans le dispositif narratif du récit de voyage, Hedin aménage une dimension théâtrale: il double le récit des événements d'un commentaire tour à tour implicite et explicite. Le narrateur s'arroge ainsi en quelque sorte le rôle de récitant. Tous les événements de Lo gsar sont rapportés dans un chapitre intitulé "Mascarades et symbolisme religieux». Hedin conclut bien à des « mascarades d'un symbolisme effrayant » lorsqu'il explique :

les lamas sont gens fort habiles et fins psychologues. Pour assurer leur prestige sur le peuple, ils s'efforcent de frapper son imagination par la magnificence et l'étrangeté des pompes religieuses et en même temps de l'amuser par des spectacles profanes adaptés à sa mentalité. (ibid., p. 118)

Ainsi, malgré une évidente sympathie affichée en chemin lors de ses rencontres avec les Tibétains, Sven Hedin reste tributaire de la conception de Pavie : il continue à voir dans la religion tibétaine une machine mise sur pied par les lamas pour maintenir le peuple en leur pouvoir. En définitive, Hedin n'analyse guère les aspects complexes d'une organisation sociale de type médiéval, mais fustige un "milieu moyenâgeux ». L'ethos de l'explorateur clairement mise en œuvre par Hedin est de «biffer la légende »: en se donnant comme but de dresser la carte du pays entier, de lever le 
mystère du système lacustre du Tibet et de résoudre le mystère des sources de l'Indus (Forêt 2004), Hedin relègue à un rang inférieur la culture tibétaine. Le « lamaïsme » est une insoutenable "vision du Moyen Âge monacal au XXe siècle ", un vestige des vieilles «fables » dont il appartient à la science occidentale de déjouer les « mystères ».

\section{Le « mystère des origines »: les danses sacrées ou l'avant-scène du drame de la modernité (Labrang, juin 1908)}

Derniers Barbares d'Henri d'ollone traite des faits religieux d'une manière un peu différente et les insère dans une problématique différente de celle de Hedin. D'ollone parcourt entre 1907 et 1908 le « mince ruban de terre fertile entre les déserts du Tibet et de la Mongolie, et unique trait d'union entre la Chine et le monde occidental ». Une fois parvenu en territoire à dominance culturelle tibétaine, l'explorateur se familiarise avec les lamas et les sanctuaires bouddhiques qui parsèment son itinéraire jusqu'au monastère de Labrang (Bla brang). Là, il assiste à l'improviste, et primus inter pares, assure-t-il, à des danses sacrées, "cérémonie tout à fait étrange ». Il nomme les instruments de l'orchestre en des termes particulièrement hétéroclites: "des trompettes de toutes les tailles - quelques-unes dépassant un mètre, - des hautbois, des conques, des tam-tams, des cymbales " (D’ollone 1911, p. 334). Il décrit les officiants selon des systèmes de référence tout aussi éclectiques. Des comparaisons quelque peu saugrenues servent à accentuer les impressions hétéroclites du voyageur :

Tout autour de la terrasse qui s'étend devant le temple, formant un vaste demicercle, une trentaine de jeunes lévites exécutaient des danses sacrées. Elles se composaient de pas rythmés, accompagnés de balancements du buste ; les bras nus, étendus dans le prolongement des épaules, soutenaient l'excédent d'étoffe que présente par en haut la pièce de tissu rouge drapée qui constitue le vêtement monacal, et figuraient très exactement des ailes de chauve-souris. (ibid., p. 335)

Enfin, en multipliant les incises, il tente de saisir la pulsation de la représentation :

Pour que leur légèreté fût plus grande, les danseurs étaient nu-pieds, leurs bottes posées à côté d'eux. Les pas étaient tantôt lents et harmonieux, tantôt rapides, accompagnés de voltes et de bonds, mais toujours avec un rythme et un ensemble parfaits. Enfin, après une cadence vive, violente, presque frénétique, musique et danses s'arrêtèrent brusquement. (ibid., p. 335)

Regrettant de ne pouvoir photographier la scène dans de meilleures conditions d'éclairage, d'ollone substitue à l'évidence de l'image photographique un commentaire que l'on peut lire comme une critique théâtrale : « ces danses n'ont rien de commun avec les scènes carnavalesques, tant de fois décrites, par lesquelles les lamas figurent la défaite des démons: elles sont véritablement gracieuses et nobles» (ibid., p. 336). De manière alors véritablement originale, d'ollone fait l'apologie des danses 'cham tibétaines et leur reconnaît des qualités artistiques. Son appréciation dramaturgique, sensible au rythme du spectacle et à l'impression d'ensemble, se démarque remarquablement de l'axiologie antithéâtrale de Hedin.

Il ne demeure pas moins que les séquences de la description attestent la difficulté à dire ces danses sacrées et à les inscrire dans un système de référence stable. D'Ollone conclut ainsi : 
Les anciennes religions, aussi bien l'hébraïque que la grecque ou l'égyptienne, comprenaient toutes des danses dans leurs cérémonies ; mais je crois bien que, mis à part les derviches tourneurs, il n'y a point d'autre exemple connu, à l'heure actuelle, de danses sacrées. (ibid., p. 336) contemporaine (Bajac 1999), renvoie à un passé bien antérieur au Moyen Âge de Hedin : mystères grecs, cérémonies égyptiennes, rites hébraïques. L'évocation des "lévites " enjoint en particulier de lire cette description sous un angle quelque peu allégorique. Le «Lha-brang»-d'ollone prend à tort le «bla» (comme dans «lama») pour «lha » (dieu) - devient notamment une allégorie du Temple de Jérusalem. Plus loin, il comparera le Wutai shan au " Mont Salvat » et la musique tibétaine (un «prodige », ditil) au «motif du Graal » du Parsifal de Wagner (D'ollone 1911, p. 351) ${ }^{11}$ ? Toujours est-il que la rencontre avec les lamas et les pratiques religieuses des Tibétains suscite dans le récit de d'ollone un élan mystique devant la foi de ces barbares prisonniers de ce désert de montagnes sauvages et appelle de vertigineuses comparaisons transculturelles. l'avant-scène d'un drame qui les englobe et les dépasse. Un épisode ultérieur permet de saisir toutes les implications de ce "mystère" tibétain. Le récit de d'ollone (son " apothéose ») culmine dans "une vision synthétique de ce monde étrange » (ibid., p. 370) que lui offre la rencontre au Wutai shan avec le dalaï-lama (Sperling 2011). Il assiste au départ du pontife pour Pékin, fin juillet 1908: «le Bouddha vivant et ses montagnards s'en allaient tout uniment monter en chemin de fer, sans rien perdre, eux, de leur rudesse, lui, de sa divinité ». Figure moderne de l'exil, cette vision est aussi un « mystère » primordial qui se joue devant les yeux de l'explorateur :

Si immense, si divers est ce monde des Barbares, que chaque découverte ne fait que mieux mesurer la profondeur de notre ignorance. Mais, d'y avoir pénétré, nous gardons en notre cœur un peu de cette horreur sacrée que la révélation des mystères causait jadis aux initiés : il nous semble qu'un coin de voile s'est soulevé, nous laissant deviner dans l'ombre quelque chose du passé qui survit et de l'avenir qui se prépare. (D'Ollone 1911, p. 371)

Précédemment, d'ollone insérait la scène tibétaine dans la perspective comparatiste de l'histoire des religions : en ethnographe, il pense trouver à Labrang un cas inédit et inconnu de danse sacrée; en ethnologue, un chaînon manquant dans l'histoire de l'humanité. En décrivant le monde tibétain, d'ollone déploie des références culturelles qui assimilent les drames sacrés tibétains à la "légende » wagnérienne, aux rites hébreux, nous l'avons vu, mais aussi, ici, aux cultes à mystères antiques. La dimension du «mystère ", sous-jacente dans les descriptions que nous avons lues, devient explicite dans la fin du récit du voyage. Contrairement à Hedin, la notion de "mystère » renvoie chez d'ollone moins au genre dramatique médiéval et à l'ordre de la mise en scène que, dans le sillage de la seconde étymologie ("vérité secrète »), à une pure vision débordant toutes les limites du cadre de représentation. S'il n'est pas soumis au soupçon critique, le mysterium tremendum se résout ici dans un syncrétisme mystique, où les deux mondes en confrontation - moderne et profane vs. primitif et sacré - se rejoignent moyennant le report de l'axe diachronique sur l'axe synchronique : « Monde primitif, violent, barbare, en un mot, distant du nôtre de quinze siècles, mais déjà tout proche dans l'espace » (ibid., p. 387). La rencontre de d'ollone avec le Tibet inconnu lui présente une "scène primitive » : par cette hiérophanie, l'avenir du monde moderne 
trouve une voie de salut en s'harmonisant avec le patrimoine immémorial de l'humanité.

\section{Un spectateur désorienté. L'outre-scène du drame sacré : au-delà du lamaïsme (Gata, 25 juillet 1909 et Tchangou, 17 août 1909)}

« Mystère » orchestré par les lamas chez Hedin, « mystère » des origines chez d'ollone, les manifestations rituelles tibétaines suscitent tour à tour des appréciations critique et apologétique. Ainsi les pratiques rituelles vues à travers le "prisme" théâtral se doublent-elles de significations secondes où la démarche ethnographique rencontre autant les hypothèses ethnologiques que les $a$ priori idéologiques. Hedin y voit une forme primitive et dégradée et considère le "mystère" tibétain, marqué d'une différence irréductible, comme un repoussoir. D'ollone, quant à lui, y voit une voie d'accès aux temps primordiaux de l'humanité - partant, à son propre inconscient. En cela, il tend à nier toute différence culturelle, les particularités ethnographiques des danses sacrées s'abîmant dans le fonds commun à tous les hommes. Le "mystère " qu'inspirent les rituels tibétains aux voyageurs ne révélerait-il donc que les limites de leur regard ? La " découverte " n'est-elle qu'une illusion cognitive et culturelle ? Ces questions ont été posées à la même époque par Jacques Bacot, qui déclarait - non sans une allusion maligne aux vastes étendues que Hedin avait arpentées ou aux milliers de kilomètres avalés par son contemporain d'ollone - avoir « recherché les hommes, ces Tibétains méconnus » plutôt que « les vastes étendues glacées qu'on parcourt pendant des mois sans voir âme qui vive » (Bacot 1909, pp. i-ii). C'est précisément en compagnie de Tibétains uniquement qu'il parcourra le monde tibétain du Kham (Khams), à la frontière entre le Tibet et la Chine.

Il raconte avoir été le premier Européen à entreprendre le pèlerinage de la montagne sacrée Kawakarpo (Kha ba dkar po) (Bacot 1909). Il rapporte les récits mythologiques sur la montagne du «cavalier blanc »; il prête attention aux pratiques dévotionnelles des pèlerins et il cerne leur rapport à l'espace : « Le feu, de cèdre et de cyprès, répand dans la forêt l'odeur des sanctuaires" (ibid., p.133). Ce faisant, Bacot fait preuve d'une attention ethnographique radicalement différente du regard lourd de préjugés de ses prédécesseurs sur les rituels des Tibétains. Lors de son second voyage (Bacot 1912), la description des pratiques religieuses est devenue, dès les premières pages, un enjeu de première importance. Comme d'ollone, il assiste à des «danses sacrées " jouées sur le parvis du temple du monastère de Gata (mGar thar). À son tour, Bacot compare le drame sacré au théâtre sacré occidental. La représentation se rapproche notamment du théâtre antique, car «le dialogue est à la fois chanté et dansé. Après chaque phase du drame, les acteurs se réunissent et lisent le récit. Après la forme trilogique, voilà aussi le chœur du drame antique» (Bacot 1912, p. 24). Le drame rappelle de surcroît les «mystères» du Moyen Âge par son sujet religieux (une vie de Bouddha), par les circonstances de l'exécution (" une ville de tentes s'est formée», "pendant trois jours », dans la "première enceinte » de la lamaserie) et par l'alternance structurelle des genres:

Ces légendes sacrées, jouées par des moines sur le parvis du temple, me rappellent

les mystères chrétiens. À certains moments, des pitreries font rire les spectateurs, 
des pitreries anciennes et vénérables, tradition d'un passé à jamais inconnu. (ibid., p. 25) théâtre tibétain : à son retour de voyage, il traduira le texte du drame vu à Gata : le Chos rgyal Dri med kun ldan gye rnam thar, version tibétaine dialoguée du Vessantara Jātaka ${ }^{13}$ (Bacot 1914). Le célèbre indianiste Sylvain Lévi, lui-même pionnier des études théâtrales dans le monde indien (Lévi 1890), assurera la direction de ce travail, indiquant la sensibilité dramaturgique des études indiennes et tibétaines françaises de cette période. Sur une plus longue durée, les répercussions de la rencontre inaugurale de Bacot avec le théâtre tibétain se font sentir à travers ses publications: les Trois mystères tibétains en $1921^{14}$, Zugiñima en 1957. L'expérience théâtrale de Gata ouvrait au futur philologue, ainsi qu'à la tibétologie moderne, un champ d'études inattendu (Thévoz 2012).

mois après son passage à Gata, Bacot est, comme Hedin avant lui, le témoin d'une séance de possession que d'aucuns de ses contemporains qualifieraient d'horrifique. À Tchangou (Brag 'go), il assiste à une séance de possession d'un « lama medium » (Bacot 1912, p. 332) ou "Kotupa » (sku rten pa) (Nebesky-Wojkowitz [1956] 1996, pp. 409-443). Là où son prédécesseur n'aurait vu que "mascarade ", Bacot est amené à s'interroger sur les conceptions tibétaines relatives à « l'incarnation d'un dieu protecteur dans le corps d'un lama » (Bacot 1912, p. 44). Pareille remise en question lui fait « éprouve[r] le malaise de se trouver sur le seuil d'un monde redoutable et inconnu » (ibid., p. 50). Tout comme Hedin, Bacot est attentif à l'orchestration: "Toute cette ville bourdonne de prières, immense orchestre de voix humaines, de cymbales, de gongs, de cloches » (ibid., p. 41). Lui aussi en éprouve les effets sensoriels puissants : "Je ne sais rien qui fixe la pensée et l'hypnotise autant que cette musique martelante, inexorable, obsédante jusqu'à en devenir pénible »(ibid.). À l'inverse de l'explorateur suédois, Bacot ressort profondément ébranlé de son expérience des rituels tibétains :

Les rites du lamaïsme tibétain sont les seuls qui troublent toujours ma curiosité blasée. Aucune autre religion ne frappe aussi violemment le spectateur le plus indifférent, ne jette ainsi l'homme hors de sa sphère familière, dans ce trouble vague qui est le seuil de l'inconnaissable. Ces prières obstinées, ces voix chaudes 
comme le parfum de l'encens, donnent la sensation que des dieux les écoutent. Chez les Tibétains la religion est un très grand art. (ibid., p. 42) caractérisait les descriptions de Hedin et de d'ollone. Pourtant, les différences sont d'emblée perceptibles dans le ton de la description et l'attention avec laquelle Bacot cherche à rendre, sans l'expliquer, ce moment intense. Ne pas comprendre n'implique ni de se désintéresser bonnement et simplement des pratiques religieuses ni de projeter ses propres schèmes culturels sur les formes rituelles découvertes. Au contraire, l'incompréhension initiale devient le moteur même de l'exploration. Ainsi, dès la brève exposition de ce que son compagnon Adjroup Gumbo lui a dit du drame, se distingue nettement un va-et-vient entre la sphère culturelle du narrateur (et de son lecteur) et celle des Tibétains :

17 août. - Dès huit heures j'étais à la lamaserie avec des soldats. En ce jour, le deuxième de la lune, aura lieu l'incarnation d'un dieu protecteur dans le corps d'un lama. Celui-ci, « le Kotupa » est un frère lai des plus humbles. Le deuxième jour de chaque lune il perd connaissance d'une façon naturelle ou provoquée, et le lendemain n'a aucun souvenir de la cérémonie extraordinaire qu'il célèbre. (ibid., p. 44)

La superposition des calendriers est révélatrice de l'espace interculturel dans lequel Bacot pénètre alors. Dans la même perspective, l'on relèvera les termes tibétains mis entre guillemets ou non, le caractère médiatif de l'information («aura lieu...»), la suspension du jugement à propos d'un phénomène inexplicable. Le voyageur, placé devant l'inconnu, traduit tout au long de la description de la séance de possession la dimension phénoménologique de l'expérience : son attention est littéralement tendue vers ce qui se passe, en termes dramaturgiques, « hors scène ":

Un rideau voile, à mi-hauteur, l'ouverture de la porte. Mais, d'une sorte de tribune, en face, dans le vestibule, on voit par-dessus le voile tout l'intérieur du sanctuaire. Après l'office, on prélude au mystère terrible qui va suivre [...].

La porte du temple est ouverte. Un rideau de soie grise et molle voile la moitié inférieure de l'ouverture noire. (ibid., pp. 45-47)

Le voile joue le rôle de seuil, séparant les espaces du visible et de l'invisible, par-delà lequel le spectateur profane ne peut qu'entrevoir la scène sacrée. Car c'est bien au-delà de ce seuil que se déroule le «mystère » dont Bacot décrit la disposition, le protocole, les gestes rituels des moines. Le voyageur éprouve le drame comme une expérience totale: des sons, des odeurs envahissent l'observateur; l'air, dit-il, se remplit progressivement de sons effroyables et de "voix de catacombes", de l'odeur des feuillages odorants et de leur fumée blanche. Ces sensations éprouvées « font vibrer le squelette dans la chair» (ibid., p. 46). L'expérience du «mystère» se situe véritablement aux franges du monde ordinaire et transgresse les limites de la scène visible: «Des cymbales déchirantes clament dans la nuit du temple que les trompes funèbres font encore plus noire et plus creuse. Le mystère s'accomplit dans ces ténèbres " (ibid., p. 48). Pour faire sentir à son lecteur ce qui se joue " hors-scène ", en deçà du visible, au moment de la crise dramatique, Bacot emploie d'ailleurs le procédé dramaturgique de la téichoscopie. Cette technique, développée dans le théâtre grec et affectionnée des Symbolistes, permet par un récit au présent de « faire voir à travers le mur » l'irreprésentable sur scène :

L'esprit du lama quitte son corps où va rentrer la divinité. Ce qui marche maintenant, ce qui avance parmi ces majestueux rugissements de bronze est un cadavre animé par un dieu. (ibid., p. 47)

Études mongoles et sibériennes, centrasiatiques et tibétaines, 46 | 2015 
41 Au paroxysme du cérémonial, la scène sacrée se dévoile littéralement : « Le voile de la grande porte s'est affaissé sur le seuil ». Si le spectateur transgresse le seuil par la vue, c'est surtout par l'imagination qu'il accède au sens du « mystère », qu'il adopte le point de vue des participants et se glisse le temps de l'événement dans leur peau, guidé par les avertissements que lui ont fournis ses compagnons de route. Ainsi la mise en scène dramaturgique ne se réduit-elle pas ici à la mise en scène d'une « mascarade ». Bien au contraire, le voyageur reconnaît que le drame ne se réduit pas aux limites de ce qui, sur scène, s'offre au regard de l'observateur extérieur :

Le dieu incarné est là, immobile et terrifiant. Son visage grimace de colère et de souffrance; ses yeux suintent de sang et ses lèvres dégouttent de bave. [...] Il est tout secoué de chocs intérieurs comme si la divinité prisonnière bouillonnait de rage dans ce corps trop petit, boursouflant les muscles, la face et les yeux qui perlent de sang. (ibid., p. 49)

Tout l'intérêt de la description, me semble-t-il, est d'osciller du début à la fin entre deux interprétations. La première voudrait qu'on abuse de la crédulité du spectateur (la religion comme un "très grand art »). La seconde suppose que quelque chose de bien plus fondamental soit à l'œuvre ici, qui justifie la patiente et scrupuleuse description de la scène qui s'étend sur plus de dix pages. Le « mystère » qui se joue devant ses yeux ne laisse pas intact même le spectateur étranger : ainsi se fondent chez Bacot les deux étymologies du terme, où la forme dramatique, évoquée par lui sous un rapport heuristique à des fins ethnographiques, entre en résonance avec la perception d'une «vérité » anthropologique qui ne nie pas les différences culturelles. La référence aux " mystères » médiévaux et au théâtre antique échappe à la simple tonalité exotique qu'elle avait chez Hedin et prend son sens plein en tant que mode théâtral participatif d'ordre sacré, tel, du moins, que l'on se l'imagine alors. Le voyageur n'assiste plus à un spectacle, au sens classique du terme, où proscenium, loges et parterre sont clairement distincts, comme chez Hedin où le «mystère" s'insérait dans un dispositif d'observation. Au contraire, Bacot est immergé dans le drame : arrière-scène, avantscène et enceinte communiquent au point que le dispositif scénographique s'efface et où visible et invisible s'interpénètrent. Alors que Hedin prétendait accéder aux coulisses de la scène $e^{15}$, Bacot, après avoir initialement souligné les relations du lieu théâtral avec ce qui l'entoure en termes spatiaux et intersubjectifs, se concentre sur la manière dont l'espace scénique advient à la conscience du spectateur et se fait ainsi signe pour la communauté réunie. Aussi la conception d'une «outre-scène » n'a-t-elle que peu en commun avec le « mystère » des origines de d'ollone. Ici, la représentation $\mathrm{du}$ rituel ne procède pas par a priori. L'expérience vécue ne débouche qu'a posteriori sur une tentative d'explication :

Son cas a sans doute un nom scientifique. Mais il y avait autre chose, quelque chose d'inexprimable qui n'est pas entièrement humain. On éprouvait le malaise de se trouver sur le seuil d'un monde redoutable et inconnu. Les Tibétains sont un peuple étrange qui vit à part des autres et ne fait rien comme eux. Après tout, nos laboratoires pourraient bien n'être pas l'Univers. (ibid., pp. 49-50)

Plutôt que de réduire le phénomène à un système théorique clos (« sans doute un nom scientifique »), Bacot ouvre les possibles interprétatifs. On perçoit ainsi une allusion aux expériences et sciences dites "psychiques ", phénomène fin de siècle qui émouvait les contemporains du voyageur à l'heure des leçons du $\mathrm{D}^{\mathrm{r}}$ Jean-Martin Charcot, des conférences d'Henri Bergson et de la modélisation de la première topique par Sigmund Freud. Cependant, Bacot, ici comme ailleurs, en avançant qu'«après tout, nos 
laboratoires pourraient bien n'être pas l'Univers ", ne fait pas prévaloir d'explication préconstruite: aussi les recherches en sciences de l'esprit du tournant du siècle n'absorbent-elles pas à elles seules le champ de questionnement qu'il découvre. Par exemple, l'anthropologie et la sociologie naissantes prêtent également attention aux phénomènes de possession ${ }^{16}$. Cependant, la réflexion de Bacot n'est que lointainement sociologique, c'est un autre courant de pensée, issu de la philosophie allemande dans la veine des Geisteswissenschaften, que l'on peut évoquer. En effet, dans la phénoménologie religieuse de Rudolf Otto, l'essence de la religion serait contenue dans l'expérience du sacré et la définition que Otto donne de la notion d'« horreur sacrée » (Otto [1917] 2001, p. 40, cité supra) est remarquablement proche de la conception de Bacot (Bacot 1912, p. 42), où le mysterium tremendum se situe au-delà de l'entendement et de la «sphère familière », tandis que d'ollone s'en tenait à une formule archaïsante et littéraire.

D'autres travaux contemporains sont encore plus proches de l'environnement intellectuel du voyageur. En mettant l'accent, à travers son usage de la notion de " mystère ", sur la dimension symbolique des rituels tibétains, Bacot rejoint la réflexion de Marcel Mauss sur les faits de culture (Tarot 1999, 2003); en insistant sur l'idée de participation, Bacot se rapproche encore des travaux de Lucien Lévy-Bruhl et de la notion d'« affectivité ". À sa manière, Jacques Bacot saisit les enjeux des remises en question épistémologiques de ses contemporains par une mise en suspens métaréflexive de ses propres catégories logiques. En s'interrogeant sur le sacré à travers les pratiques rituelles tibétaines - dans les deux exemples donnés ici, comme à propos, par exemple, de la tradition des «terres cachées" (sbas yul) qui donne son sous-titre à son récit (Vers Népémakö, terre promise des Tibétains) -, Bacot ouvre à la tibétologie des questionnaires nouveaux, connexes aux réflexions des fondateurs des sciences humaines modernes. Ce faisant, il dégage les «études thibétaines » de l'orbe du lamaïsme et invite à comprendre dans sa complexité et pour elle-même ce que Rolf Stein appellera plus tard la « civilisation tibétaine».

\section{Le theatrum mundi d'une orientaliste bouddhiste : simulacre lamaïque et illusio bouddhique (Lachen, mai 1912)}

À une poignée d'années près, Alexandra David-Néel rencontre les formes du bouddhisme tibétain au Sikkim en avril 1912. Mais avant même d'avoir pénétré au Tibet, David-Néel se déclarait « bouddhiste pratiquante et militante » (David-Néel 1975, p. 109) : à Kalimpong (Kālimpong), elle explique au dalaï-lama stupéfait qu'elle l'est devenue non pas en Asie, mais à Paris, « sur les bancs d'une université européenne en étudiant la philosophie orientale » (ibid., p. 124). La position originale, mais ambiguë, de l'exploratrice face aux rituels tibétains illustre une attitude qui, bien qu'on y reconnaisse en creux l'empreinte, se démarque de celle de ses prédécesseurs. C'est en ce sens qu'il est instructif ici de s'intéresser aux lettres qu'elle écrit à son mari lors de son séjour en Asie. Dans la lettre du 30 mai 1912, elle raconte la cérémonie à laquelle elle a assisté au monastère de "Lachen ». Cette lettre est exemplaire, car nous y retrouvons comme une synthèse des approches des voyageurs précédents. David-Néel souligne pour commencer le caractère exotique de la scène :

À l'entrée du monastère, tous les lamas rouges, le grand lama supérieur en tête, nous reçoivent. Les musiciens, les porteurs d'ombrelles, de bannières etc. sont 
rangés en haie. Il y en a qui soufflent dans des trompettes tibétaines si longues que le bout en est appuyé à terre. C'est extraordinairement pittoresque. (ibid., p. 156) formée aux études bouddhiques par Philippe Édouard Foucaux, Léon Féer et Sylvain Lévi au sein des cercles savants français. Depuis les travaux fondateurs d'Eugène Burnouf, ces études étaient centrées sur le bouddhisme dit du Nord, généralement considéré comme dégénéré, comme le soutenait Pavie, car ultérieur aux premiers développement du bouddhisme en Inde. Cependant, David-Néel a aussi, par ses voyages, fréquenté les spécialistes allemands et anglais comme Hermann oldenberg et Thomas W. Rhys Davids. Contrairement à l'école française (Lévi [1924] 1937), ces derniers ont mis l'accent sur le bouddhisme du Sud, dit orthodoxe. Accédant aux enseignements du bouddhisme par les textes, David-Néel privilégie, conformément à la tradition philologique, les témoins les plus anciens, supposément plus proches de la doctrine du Bouddha lui-même. Sa conception des rituels tibétains respecte en ce sens - et bien qu'elle se défende d'appartenir à ces « érudits ferrés de racines grammaticales » (DavidNéel 1975, p. 109) - les sentiments alors largement partagés sur le lamaïsme :

Les lamas chantent alors la formule du "triple refuge ", cela est en tibétain et, avec ce chœur, bien différent de la récitation à Ceylan. [...] Voilà bien du ritualisme... Enfin pour l'amour du pittoresque, résignons-nous. [...] Oh! Je me les rappellerai souvent au moment de faire des conférences en Europe, ces discours dans ces milieux exotiques, parmi les idoles extravagantes, les symboles effarants et l'auditoire fantastique des lamas rouges. Puis le supérieur se place dans la nef centrale ayant retiré sa mitre. (ibid., p. 155)

Ce "ritualisme " outrancier, orchestré par les lamas, est loin de gagner l'intérêt de celle qui sera plus tard la célèbre vulgarisatrice du bouddhisme tibétain : «C'est long, long, interminable, entrecoupé de multiples prosternations. [...] Enfin, c'est terminé » (ibid., p. 158). En contrepartie, c'est la voyageuse elle-même qui dispensera à Gangtok (lettre du 23 juin) le message authentique du bouddhisme : « dans l'oratoire tantrique, je parle de la grande doctrine que ces lamaïstes ont oubliée ou qu'ils se contentent de connaître égoïstement, laissant la foule dans sa superstition grossière » (ibid., p. 164). Aussi assure-t-elle à son mari qu'elle est reconnue comme une autorité auprès des lettrés de Gangtok :

On dit beaucoup de mal des lamaïstes et leur ignorance le mérite ; mais ne font-ils pas preuve d'une belle largeur d'esprit en m'appelant moi, de croyances si différentes des leurs et prêchant contre leurs superstitions, à parler dans leurs temples? (ibid., p. 173) 
lamaïsme est une religion terrifique » (ibid., p. 194). Sur le même sujet, au monastère de Toumbong, elle renchérit :

Nous ne comprenons la danse que comme évocation lascive et, comme telle, exécutée par des femmes. Mais, ici, la danse est une pantomime. Les guerriers évoluent autour de Mahâ Kala et du génie du Kintjindjinga, tour à tour apaisés et en furie. En fait, ce sont des danses sacrées qui se déroulent devant le temple... (ibid., p. 198)

51 Première chanteuse à l'Opéra-Comique d'Hanoi, David-Néel avait interprété quelques années auparavant Lakmé de Léo Delibes. Drame lyrique célèbre notamment pour l'exotisme du ballet des bayadères de l'Acte II. Aussi ne manque-t-elle pas d'y comparer les danses des lamas et d'en souligner le contraste: ces "danses sont loin d'être grotesques comme on les proclame, elles me font penser à celles des guerriers scandinaves. " Il n'en demeure pas moins que la perception de ces «danses sacrées » s'insère dans un raisonnement bien différent de celui de d'ollone quelques années auparavant. Ce qui compte aux yeux de David-Néel est d'en évaluer l'orthodoxie. Or il serait erroné de leur attribuer une quelconque vérité doctrinale et d'en justifier la pratique :

Le vieux maharadjah [du Sikkim] n'a aucune espèce de piété bouddhiste, il craint les mauvais esprits, a installé Padmasambhava sur l'autel de son sanctuaire et est un chasseur enragé. Ce qu'on chante pendant les danses est une fois de plus terrifique et horrifique comme tout ce lamaïsme qui n'est que magie et sorcellerie. (ibid., p. 198)

David-Néel est, en 1912, fortement tributaire du point de vue négatif sur le bouddhisme tibétain comme ritualisme abject. Le lecteur le sait par les récits et romans d'aventure ultérieurs qui la rendront célèbre, l'auteur atténuera sa position initiale au fur et à mesure qu'elle se familiarisera avec le bouddhisme tantrique. Ce jugement initial ne disparaîtra pourtant pas entièrement dans le récit de ces mêmes expériences qu'elle racontera au grand public dans Mystiques et Magiciens du Tibet en 1929, ouvrage rédigé dans la lancée du Voyage d'une Parisienne à Lhassa en 1927. Revenant en effet dix-sept années plus tard sur ces événements, elle fait alors état d'un changement de point de vue. Dans sa correspondance avec son mari, la «névrose » des pratiquants du «chöd » $(g c o d)^{17}$ ne manquait pas de la laisser sceptique. Dans ses récits destinés au grand public, elle présente ce même rituel tantrique comme une variante orthodoxe des différentes "méthodes d'atteindre tharpa, de se libérer complètement de l'illusion, d'effacer le mirage du monde imaginaire et d'affranchir son esprit des croyances chimériques " (David-Néel 1929, p. 165). C'est là le bouddhisme « secret » qu'elle dissocie si nettement de ce qu'elle continuera d'appeler, en 1953, le « lamaïsme $»^{18}$.

53 La notion de "mystère ", chez David-Néel, sert donc encore à comprendre les rituels tibétains, mais elle se partage entre ses deux acceptions. Elle désigne un "bric-à-brac » « effarant » quand elle se rapporte d'abord, comme chez Hedin, aux « offices » du clergé tibétain, gestes sans signification pour les fidèles «superstitieux ». Elle ne se rapporte pas moins à une "vérité secrète ", dans un sens quasi occulte, étranger à d'ollone et à Bacot: pour David-Néel, le «mystère» donne alors la possibilité d'atteindre le « domaine de l'Autre. » En Inde, le «mystère » se jouait derrière le « rideau symbolique du Temple de Chidambaram » $(1975, \text { p. } 78)^{19}$ :

Innommable trompette... innommable musique de trois notes répétées à l'octave, avec des quintes qui sonnent crues, dures, infernales, emplissent le temple d'horreur. Ah! Comment décrire cette vision, le frisson qu'elle vous fait courir par les moelles! On est à côté de la terre, dans le monde des influences terribles et 
mauvaises, le domaine de "l'Autre » comme on disait au Moyen-Âge. Et réellement on le sent passer sur soi le souffle de «l'Autre». Et puis les portes illuminées des sanctuaires interdits qui s'ouvrent sur des gouffres à la fois scintillants et obscurs... Quelle vision inoubliable où passent tant de choses en plus de celles que l'on voit avec ses yeux de chair. (David-Néel 1975, p. 59) ${ }^{20}$ tantrisme tibétain. Aussi les rituels tibétains sont-ils porteurs d'une "vérité secrète " s'ils se conforment à l'orthodoxie doctrinale bouddhique de "Sunyata le Grand Vide » (ibid., p. 148). Pratiqués par les initiés, seuls aptes à dépasser l'illusio de la représentation, ils conduisent à «effacer le mirage des phénomènes » et à sublimer le theatrum mundi de l'existence.

\section{Les savoirs sur les rituels : les dessous culturels et interculturels du « mystère »}

Il est remarquable que, dans la première décennie du XIX ${ }^{e}$ siècle, les voyageurs assimilent différents faits culturels tibétains au «mystère» pour en souligner la dimension rituelle. Cette congruence historique nous rappelle d'abord que toute approche, hier comme aujourd'hui, est contingente, située dans le temps et dans l'espace. Ainsi est mis en lumière un moment-pivot dans l'histoire des représentations et des savoirs sur les rituels au Tibet. La notion de lamaïsme est encore active quand elle est projetée sur la culture tibétaine (Hedin pour un jugement négatif, d'ollone pour une appréciation positive). Toutefois, le «ritualisme» tibétain prend un visage nouveau, lorsque naissent de nouveaux critères épistémiques: ainsi l'approche de Bacot fait écho au renouvellement des questionnements des sciences humaines, tandis que David-Néel oppose au «lamaïsme » des rituels d'ordre privés, dont la dimension spirituelle s'intègre dans la vision du bouddhisme à laquelle elle adhère.

Cependant, ces questions d'ordre épistémologiques ne doivent pas faire oublier que, par delà la diversité des formes rituelles rencontrées, le "prisme " théâtral et la référence au «mystère » agissent comme un modèle d'intelligibilité commun à chacun des voyageurs, ce à défaut d'une discrimination avertie entre les diverses formes cultuelles et théâtrales tibétaines ou d'une connaissance des nomenclatures et typologies indigènes. Ces dernières paraîtront aujourd'hui, peu ou prou, au regard de l'ethnographe averti ; l'ethnohistorien, quant à lui, les décèlera, a posteriori, dans les textes des voyageurs.

Cette déficience transitoire ne fournit pourtant pas une explication pleinement satisfaisante. J'ai souligné combien la notion de «mystère " revêt des significations profondes aux yeux des explorateurs dans le contexte des années 1910 ; celle-ci désigne certes une forme dramatique historique, mais elle incarne avant tout à cette période une idée de la représentation théâtrale. J'ai alors cherché à cerner la complexité que sa récurrence remarquable, unanime mais non uniforme, suppose alors. Analyser ces significations permet d'apercevoir les valeurs parfois opposées que le "mystère » représente pour les voyageurs. Symétriquement, les usages contrastés que ceux-ci lui réservent ouvrent sur des modes de connaissance singuliers.

On observe ainsi des actualisations plurielles de la notion de «mystère ». Les voyageurs y associent d'autres formes dramatiques : théâtre classique chez Hedin, théâtre antique et rites hébraïques chez d'ollone, théâtralité symboliste chez Bacot, spectacle exotique

Études mongoles et sibériennes, centrasiatiques et tibétaines, 46 | 2015 
chez David-Néel, la référence wagnérienne omniprésente. Ils lui confèrent aussi différents enjeux métathéâtraux: "mascarade » chez Hedin, "tableau vivant » chez d'ollone, «participation » et « régime symbolique » chez Bacot, illusio et theatrum mundi chez David-Néel. Cette pluralité de significations masquée par l'univocité de la référence au «mystère » conduit ainsi à considérer les approches des rituels tibétains sous un double rapport : tout en renvoyant à la culture propre des voyageurs, la notion de «mystère » ne révèle pas moins les situations d'expérience qu'elle met en œuvre dans la rencontre interculturelle.

\section{BIBLIOGRAPHY}

App, U.

2011 Richard Wagner and Buddhism (Rorschach/Kyoto, UniversityMedia).

2012 The Cult of Emptiness. The Western Discovery of Buddhist Thought and the Invention of Oriental Philosophy (Rorschach/Kyoto, UniversityMedia).

Arvon, $\mathrm{H}$.

1951 Le Bouddhisme (Paris, PUF).

Bacot, J.

1909 Dans les Marches tibétaines. Autour du Dokerla (novembre 1906-janvier 1908) (Paris, Plon-Nourrit). 1912 Le Tibet révolté. Vers Népémakö, la Terre promise des Tibétains (Paris, Hachette).

1914 Drimekunden. Une version tibétaine du Vessantara Jataka, Journal asiatique, IX/4, pp. 221-305.

1921 Représentations théâtrales dans les monastères du Thibet. Trois mystères tibétains :

Tchrimekundan-Djroazanmo-Nansal (Paris, Bossard).

1957 Zugiñima (Paris, Cahiers de la Société asiatique).

Bajac, Q.

1999 Tableaux vivants. Fantaisies photographiques victoriennes (1840-1880) (Paris, RMN).

Barba, E.

[1993] 2003 Le Canoë de papier. Traité d'anthropologie théâtrale (Montpellier, L’Entretemps).

Baumgartner, E.

1988 Moyen Âge, 1050-1486 (Paris, Bordas).

Bishop, P.

1989 The Myth of Shangri-La. Tibet, Travel Writing and the Western Creation of Sacred Landscape

(London, Athlone Press).

Bonoli, L. \& S. Thévoz

2012 L'Effet de différence. L'altérité culturelle dans Indian Tango d'Ananda Devi, Théorie Littérature Épistémologie, 29, pp. 71-91.

Conze, E.

1958 A Short History of Buddhism (Bombay, Chetana).

D'ollone, $\mathrm{H}$.

1911 Les Derniers Barbares. Chine-Tibet-Mongolie (mission d'ollone 1906-1909) (Paris, Pierre Lafitte). 
David-Néel, A.

1927 Voyage d'une Parisienne à Lhassa (Paris, Plon).

1929 Mystiques et Magiciens du Tibet (Paris, Plon).

1969 L'Inde où j'ai vécu (Paris, Plon).

1953 Le Vieux Tibet face à la Chine nouvelle (Paris, Plon).

1975 Journal de voyage. Lettres à son mari, 11 août 1904-27 décembre 1917, vol. I (Paris, Plon).

Droit, R.-P.

1997 Le Culte du néant. Les philosophes et le Bouddha (Paris, Le Seuil).

Folco, A.

2006 Dramaturgie de Mallarmé (thèse de doctorat sous la direction de Jean-Pierre Sarrazac, université de Paris-3, non publiée), 2 vol. , 493 p.

Forêt, P.

2004 La Véritable Histoire d'une montagne plus grande que l'Himalaya : les résultats scientifiques inattendus d'un voyage au Tibet, 1906-1908, et de la querelle du Transhimalaya (Paris, Bréal).

Gréban, A.

$\left[\mathrm{XV}^{\mathrm{e}} \mathrm{s}\right.$.] 1878 Le Mystère de la Passion, publié d'après les manuscrits de Paris, avec une introduction et un glossaire par Gaston Paris et Gaston Raynaud (Paris, Vieweg).

Gréban, A.

[1452] 1901 Le Vray Mistère de la Passion, adapté par Charles Gailly de Taurines et Léonel de la Tourasse, avant-propos par Émile Faguet (Paris, Belin frères).

Hedin, S.

1910 Le Tibet dévoilé, ouvrage traduit et adapté par Charles Rabot (Paris, Hachette).

Henrion-Dourcy, I.

2006 Ache lhamo. Jeux et enjeux d'une tradition thétrale tibétaine, Études mongoles et sibériennes, centrasiatiques et tibétaines [En ligne], 36-37, mis en ligne le 25 février 2009, consulté le 07 décembre 2013. URL : http://emscat.revues.org/1046.

Huc, R.-É.

[1850] 2001, Souvenirs d'un voyage dans la Tartarie et le Thibet, pendant les années 1845-1846 (Paris, Omnibus).

Keck, F.

2008 Lévy-Bruhl entre philosophie et anthropologie. Contradiction et participation (Paris, Éditions du CNRS).

Lalou, M.

1957 Les Religions du Tibet (Paris, PUF).

Levenson, $\mathrm{C}$.

2004 Le Bouddhisme (Paris, PUF).

Lévi, S.

1890 Le Théâtre indien (Paris, Bouillon).

[1924] 1937 Les parts respectives des nations occidentales dans les progrès de l'indianisme, in Mémorial Sylvain Lévi (Paris, Hartmann), pp. 107-117.

Lopez, D.

1979 Approaching the Numinous : Rudolf Otto and the Tibetan Tantra, Philosophy East and West, 29/4, pp. 467-476. 
Lopez, D. S.

[1998] 2003 Fascination tibétaine (Paris, Autrement).

Losco-Lena, M.

2010 La Scène symboliste (1890-1896). Pour un théâtre spectral (Grenoble, ELLUG).

Lucet, $\mathrm{S}$.

1997 Le « Théâtre en liberté » des symbolistes : dérives de l'écriture dramatique à la fin du $\mathrm{XIX}^{\mathrm{e}}$ siècle (thèse de doctorat sous la direction de J. de Palacio, université de Paris-IV, non publiée), $778 \mathrm{p}$.

Nebesky-Wojkowitz, R. de

[1956] 1996 Oracles and Demons of Tibet : The Cult and Iconography of the Tibetan Protective Deities (S'Gravenhage, Mouton).

Otto, R.

[1917] 2001 Le Sacré [Das Heilige. Über das Irrationelle in der Idee des Göttlichen und sein Verhältnis zu dem Rationalen] (Paris, Payot).

Paris, P.

1855 De la mise en scène des mystères et du mystère de la Passion : cours de littérature du moyen âge (leçon du 7 mai 1855) (Paris, Dupont).

Pavie, Th.

1847 Le Thibet et les études thibétaines, La Revue des Deux Mondes, XIX, pp. 37-58.

Pavis, P.

[1996] 2006 Dictionnaire du théâtre (Paris, Armand Colin).

Pradier, J.-M.

1996 Ethnoscénologie : la profondeur des émergences, in C. Khaznadar \& J. Duvignaud (éd.), La Scène et la Terre. Questions d'ethnoscénologie (Arles, Babel, coll. « Internationale de l'imaginaire »), pp. 13-42.

Ridoux, C.

1996 La nouvelle école de philologie romane et sa perception de la littérature médiévale, Cahiers de recherches médiévales, 2, pp. 187-198.

Rozik, E.

2002 The Roots of Theatre. Rethinking Ritual and Other Theories of Origin (Iowa, University of Iowa Press).

Sperling, E.

2011 Some Western Accounts of Encounters with the Dalai Lama at Wutai Shan, JIATS, 6, décembre 2011, pp. 394-402.

Stein, R.

[1962] 1987 La Civilisation tibétaine (Paris, L’Asiathèque).

Tarot, C.

1999 De Durkheim à Mauss, l'invention du symbolique. Sociologie et science des religions (Paris, La Découverte).

2003 Sociologie et anthropologie de Marcel Mauss (Paris, La Découverte).

Thévoz, S.

2010 Un horizon infini. Voyageurs et explorateurs français au Tibet (1846-1910) (Paris, PUPS).

2011 En aval de Shangri-La : résurgences himalayennes dans l'espace francophone contemporain, Nouvelles Études francophones, 26/2, pp. 189-204. 
2012 L'Éveil de Jacques Bacot à la tibétologie : du drame sacré de Gata aux horizons de Népémakö, Journal asiatique, 1, pp. 247-268.

2012 Des poèmes... et ils sont partis. La fiction dans les récits d'exploration au Tibet, L'Homme. Revue française d'anthropologie, 201, pp. 7-30.

Waddell, L. A.

1894 The Buddhism of Tibet, or Lamaism : with its Mystic Cults, Symbolism and Mythology and Its Relation to Indian Buddhism (London, Oriental Publishers).

White, R.

1991 The Middle Ground. Indians, Empires and Republics in the Great Lakes Region, 1650-1815

(Cambridge, Cambridge University Press).

\section{NOTES}

1. Version augmentée et remaniée en profondeur du chapitre VII de mon livre (Thévoz 2010, pp. 247-298), le présent article contribue à un projet de recherche que je mène actuellement grâce au soutien du Fonds national suisse de la recherche scientifique (PA00P1_145398), que je remercie ici. En m'invitant au séminaire « Rituels » de l'équipe «TBACT » (Tibet, Bhoutan, Aire Culturelle Tibétaine) du CRCAO (UMR8155) en juin 2013, Katia Buffetrille m'a offert une première occasion de reformuler les questions abordées ici, qu'elle en soit vivement remerciée.

2. Citons, à titre d'exemple, les travaux de Richard White (1991) sur les relations entre missionnaires et Amérindiens aux XVII-XVIII siècles.

3. Dans le sillage de l'étude que l'ethnologue autrichien René de Nebesky-Wojkowitz a consacrée à la démonologie tibétaine, les formes religieuses tibétaine peuvent de nos jours encore apparaître comme une affaire d'oracles et de démons, selon le titre de l'ouvrage ([1956] 1996). Elles appartiendraient ainsi moins à la catégorie de la religion qu'à celle de la magie. Ainsi Lopez (1979) observe-t-il que de toutes les formes d'expressions religieuses, les divinités courroucées tantriques tibétaines se rapprochent le plus de ce que Rudolf Otto ([1917] 2001), appelait le mysterium tremendum.

4. Le « mystère » est, au sens commun, « ce qui est caché, c'est-à-dire ce qui n'est pas manifeste, ce qui n'est ni conçu ni compris » (ibid., p. 37) ; dans le domaine religieux, il est « le "tout autre", ce qui nous est étranger et nous déconcerte, ce qui est absolument en dehors du domaine des choses habituelles, comprises, bien connues, et partant «familières »; c'est ce qui s'oppose à cet ordre de choses et, par là même, nous remplit de cet étonnement qui paralyse. » (ibid., pp. 57-58) 5. Rappelons avec Lopez ([1998] 2003, p. 34) que le «lamaïsme» désigne une conception de la religion tibétaine héritée du chinois «lama jiao » qui signifie littéralement « religion des lama». Parallèlement au «dao jiao» (taoïsme) et au «ru jiao» (religion des érudits, confucianisme) et pour des raisons politiques, l'empereur mandchou Qianlong, en 1775, distinguait ainsi le bouddhisme tibétain de la « religion de Bouddha » («fo jiao ») dont les lamas étaient jusqu'alors les éminents représentants à la cour impériale. De facture relativement récente, le terme apparaît à une période d'intenses contacts entre l'Empire chinois et les missionnaires européens. En dehors d'un contact direct avec le Tibet et les Tibétains, ceux-ci l'introduisent durablement dans les langues européennes, imposant ainsi une vision négative de la religion tibétaine, ecclésiale de par sa forme et superstitieuse de par ses dogmes et ses pratiques fétichistes et magiques.

6. En visant avant tout à historiciser la perception des voyageurs, je ne suis ni la voie de l'ethnoscénologie - qui, cherchant «le fonds commun de l'humanité » à travers les formes multiples d'une "théâtralisation contre l'innommable », se présente comme «l'étude dans les différentes cultures des pratiques et des comportements humains organisés (PCHSO) » (Pradier 
1996, p. 16) ni celle de l'« anthropologie théâtrale » (par exemple Barba [1993] 2003) pour laquelle toute performance théâtrale renvoie à des origines rituelles et sacrées. Lire à ce propos Rozik 2002.

7. C'est à Paulin Paris, le père de Gaston, que l'on doit une leçon au Collège de France consacrée à «la mise en scène des mystères et du mystère de la Passion» (1855). En 1878, Gaston Paris et Gaston Raynaud publient le Mystère de la Passion d'Arnoul Gréban (XV ${ }^{\mathrm{e}} \mathrm{s}$.).

8. Tant et si bien que Sophie Lucet (1997, pp. 193-339) consacre la seconde partie de sa thèse sur la dramaturgie symboliste à ce qu'elle appelle un «théâtre du "mystère" ». Voir également Folco (2006, chapitre « La légende et le mystère », pp. 230-247).

9. L'ambivalence survit de fait dans l'imaginaire collectif aux mises en garde d'un Émile Faguet, par exemple, qui précisait en 1901 dans son Avant-Propos au Vray Mistère de la Passion de Gréban (1901, p. III) : «il faut écrire mistère, comme au moyen âge, et parce qu'ils avaient raison de l'écrire ainsi : mistère venant de ministerium et non pas de musterion»).

10. Voir Thévoz (2010) pour plus de renseignements biographiques sur les voyageurs.

11. Mentionnons que le compositeur Max d'ollone est le frère de l'explorateur et, élève de Massenet, auteur de plusieurs drames lyriques où l'on retrouve l'influence de Wagner. Mais Henri d'Ollone percevait-il l'inspiration bouddhique du Parsifal ou du Tristan de Wagner, lecteur de Schopenhauer et de Burnouf (App 2011)?

12. L'on comparera avec profit la définition que donne Henrion-Dourcy (2009), spécialiste du Ache lhamo auquel appartient le «drame» décrit par Bacot: "Comme la plupart des théâtres d'Asie, il est un genre composite : à la fois drame à thématique religieuse (issue du bouddhisme mahāyāna), satire mimée, et farce paysanne, il comprend de la récitation sur un mode parlé, du chant, des percussions, de la danse et des bouffonneries improvisées, ainsi qu'un usage de masques et de costumes flamboyants, qui tranchent avec la sobriété absolue des décors (la scène est vide) et de la mise en scène. Bien qu'il ait été encouragé et financé par le gouvernement des dalaï-lamas, de grands monastères et des familles aristocratiques, c'est un théâtre avant tout populaire, et non pas réservé à une élite lettrée.»

13. Le sujet de ce «mystère » est « l'histoire de l'avant-dernière existence sur Terre de celui qui renaîtra Çakya-Muni. Vessantara « Tchrimekundan des Tibétains » est le futur Bouddha. Il traverse une vie d'épreuves remarquables qu'il s'est toutes attirées par sa passion de la charité. » (Bacot 1921, p. 19)

14. Bacot résume en quatrième de couverture sa vision du «théâtre tibétain, théâtre des grandes altitudes. Les monastères où on le joue occupent des lieux choisis, ces vallées aériennes de la Haute-Asie [...] Les monastères, la scène de ce théâtre, sont des oasis de prière dans ces déserts enchantés. [...] Comme les maisons d'une ville autour de la place publique, les tentes ouvrent leurs murs de toile sur l'espace libre de la scène. Devant ces Tibétains accourus de loin, les acteurs ne représentent pas la vie, mais leur idéal de la vie. Leur thème est l'impermanence des choses. Leur théâtre est un camp de nomades qui lui-même disparaît dès que le spectacle est terminé. »

15. Significativement, Bacot dira plus tard du théâtre tibétain qu'il ne cherche guère «à produire l'illusion par le décor et la machinerie. » (Bacot 1921, p. 15)

16. Au milieu du siècle, la "démonologie» devient en tibétologie un domaine d'études particulièrement développé. Voir Nebesky-Wojkowitz [1956] 1996 et Lalou 1957, ch. « Prophètes et démons ", pp. 76-78.

17. Technique de méditation et ensemble de rituels, dont la codification remonte au $\mathrm{XI}^{\mathrm{e}}$ siècle et revient traditionnellement à Machik Labdrön (Ma gcig Lab sgron). Par cette pratique, les adeptes, parfois surnommés les «saints fous » par les voyageurs, cherchent à «trancher » (gcod) l'ego en développant des visions dans lesquelles le corps, d'où émanent les afflictions mentales, est l'objet d'un sacrifice visant à faire reconnaître l'absence de soi. 
18. Il ne faut pas oublier que, dans le sillage de Waddell (1894), le terme a perduré au XX ${ }^{\mathrm{e}}$ siècle. Voir Lopez [1998] 2003 pour l'analyse de la fortune de ce concept dans le domaine anglo-saxon. Conze (1958) disqualifie cette catégorie. En France, dans la collection «Que sais-je?», Arvon (1951) entretient le jugement négatif porté sur le bouddhisme tibétain; ce n'est qu'en 2004 que l'ouvrage est remplacé par celui de Claude Levenson.

19. Retenu pour les premières pages de L'Inde où j'ai vécu (David-Néel 1969), ce leitmotiv apparaît de manière récurrente dans sa correspondance.

20. C'est le premier moment de la longue samâdhi (David-Néel féminise le terme) que lui a procurée «l'atmosphère psychique [de l'Asie] qui a donné le jour à ces théories » (David-Néel 1975 , p. 80). Ce sont ces dernières qui sont le cœur de sa rencontre avec le bouddhisme tibétain. Elles l'orientèrent moins vers une approche de la culture tibétaine en tant que "civilisation " (Stein 1962), dans le sens de Marcel Mauss, que vers les phénomènes psychiques qu'elle considère explicitement, en conclusion de Mystiques et Magiciens du Tibet comme ressortissant d'une étude de « psychologie».

\section{ABSTRACTS}

Early 20th century travellers focused on Tibetan rituals and gave numerous descriptions in their accounts. It is striking that they all use the notion of "mystery play" to convey intelligibility to their perception of a wide and multifarious array of rituals. By contextualizing the term and analyzing the multiple meanings and values it conveys for each of the travellers, I argue that the phenomenon implies more than a symbolic projection on the Tibetan culture and gives evidence of a set of cognitive processes activated in the intercultural encounter.

Dans la première décennie du $\mathrm{XX}^{\mathrm{e}}$ siècle, les voyageurs marquent un intérêt appuyé envers les formes rituelles tibétaines et livrent dans leurs récits de voyage ou dans leur correspondance des témoignages à caractère ethnographique. Le présent article s'interroge sur la référence permanente à la notion de « mystère » dans leurs récits. En évaluant la valeur et les significations que revêt le terme aux yeux des voyageurs, il s'agit de dépasser le simple constat d'une projection culturelle. En effet, des différents regards portés sur le religieux tibétain ressortent également différentes visions et expériences de la culture tibétaine, variables en termes axiologiques, heuristiques, phénoménologiques et réflexifs.

\section{INDEX}

Mots-clés: Tibet, rituels, théâtre, cognition, récit, voyage, interculturel

Keywords: Tibet, rituals, theatre, cognition, narrative, travel, intercultural

\section{AUTHOR}

\section{SAMUEL THÉVOZ}

Samuel Thévoz est chercheur pour le Fonds national suisse de la recherche scientifique. Ses travaux portent sur la littérature de voyage au Tibet, sur les phénomènes interculturelles en 
littérature et sur la rencontre du théâtre européen et occidental avec l'Asie bouddhique.

Ouvrage : Un horizon infini. Voyageurs et explorateurs français au Tibet (1846-1910) (Paris, PUPS, 2010).

Édition : Marie de Ujfalvy-Bourdon, Une Parisienne dans l'Himalaya (Paris, Transboréal, 2014). 